\title{
Constraints on particle acceleration in SS433/W50 from MAGIC and H.E.S.S. observations
}

\begin{abstract}
MAGIC Collaboration: M. L. Ahnen ${ }^{1}$, S. Ansoldi ${ }^{2,25}$, L. A. Antonelli ${ }^{3}$, C. Arcaro ${ }^{4}$, A. Babić5 , B. Banerjee ${ }^{6}$, P. Bangale ${ }^{7}$, U. Barres de Almeida ${ }^{7,26}$, J. A. Barrio ${ }^{8}$, J. Becerra González ${ }^{9,10,27,28}$, W. Bednarek ${ }^{11}$, E. Bernardini ${ }^{12,29}$, A. Berti ${ }^{2,30}$, B. Biasuzzi ${ }^{2}$, A. Biland ${ }^{1}$, O. Blanch ${ }^{13}$, S. Bonnefoy ${ }^{8}$, G. Bonnoli ${ }^{14}$, F. Borracci ${ }^{7}$, R. Carosi $^{14}$, A. Carosi ${ }^{3}$, A. Chatterjee ${ }^{6}$, P. Colin ${ }^{7}$, E. Colombo ${ }^{9,10}$, J. L. Contreras ${ }^{8}$, J. Cortina ${ }^{13}$, S. Covino ${ }^{3}$, P. Cumani ${ }^{13}$, P. Da Vela ${ }^{14}$, F. Dazzi ${ }^{3}$, A. De Angelis ${ }^{4}$, B. De Lotto ${ }^{2}$, E. de Oña Wilhelmi ${ }^{15}$, F. Di Pierro ${ }^{3}$, M. Doert ${ }^{16}$, A. Domínguez ${ }^{8}$, D. Dominis Prester ${ }^{5}$, D. Dorner ${ }^{17}$, M. Doro ${ }^{4}$, S. Einecke ${ }^{16}$, D. Eisenacher Glawion ${ }^{17}$, D. Elsaesser ${ }^{16}$, M. Engelkemeier $^{16}$, V. Fallah Ramazani ${ }^{18}$, A. Fernández-Barral ${ }^{13}$, D. Fidalgo ${ }^{8}$, M. V. Fonseca ${ }^{8}$, L. Font ${ }^{19}$, C. Fruck $^{7}$, D. Galindo ${ }^{20}$, R. J. García López ${ }^{9,10}$, M. Garczarczyk ${ }^{12}$, M. Gaug ${ }^{19}$, P. Giammaria ${ }^{3}$, N. Godinovic ${ }^{5}$, D. Gora ${ }^{12}$, S. Griffiths ${ }^{13}$, D. Guberman ${ }^{13}$, D. Hadasch ${ }^{21}$, A. Hahn ${ }^{7}$, T. Hassan ${ }^{13}$, M. Hayashida ${ }^{21}$, J. Herrera ${ }^{9}, 10$, J. Hose ${ }^{7}$, D. Hrupec ${ }^{5}$, G. Hughes ${ }^{1}$, K. Ishio ${ }^{7}$, Y. Konno ${ }^{21}$, H. Kubo ${ }^{21}$, J. Kushida ${ }^{21}$, D. Kuveždić ${ }^{5}$, D. Lelas ${ }^{5}$, E. Lindfors ${ }^{18}$, S. Lombardi ${ }^{3}$, F. Longo ${ }^{2,30}$, M. López ${ }^{8}$, A. López-Oramas ${ }^{13,34, \star}$, P. Majumdar ${ }^{6}$, M. Makariev ${ }^{22}$, G. Maneva ${ }^{22}$, M. Manganaro $^{9,10}$ K. Mannheim ${ }^{17}$, L. Maraschi ${ }^{3}$, M. Mariotti ${ }^{4}$, M. Martínez ${ }^{13}$, D. Mazin ${ }^{7,31}$, U. Menzel ${ }^{7}$, M. Minev ${ }^{22}$, R. Mirzoyan ${ }^{7}$, A. Moralejo ${ }^{13}$, V. Moreno ${ }^{19}$, E. Moretti ${ }^{7}$, P. Munar-Adrover ${ }^{20,35, \star}$, V. Neustroev ${ }^{18}$, A. Niedzwiecki ${ }^{11}$, M. Nievas Rosillo ${ }^{8}$, K. Nilsson $^{18,32}$, K. Nishijima $^{21}$, K. Noda $^{7}$,

L. Nogués ${ }^{13}$, S. Paiano ${ }^{4}$, J. Palacio ${ }^{13}$, D. Paneque ${ }^{7}$, R. Paoletti ${ }^{14}$, J. M. Paredes ${ }^{20}$, X. Paredes-Fortuny ${ }^{20}$, G. Pedaletti ${ }^{12}$, M. Peresano ${ }^{2}$, L. Perri ${ }^{3}$, M. Persic ${ }^{2,33}$, P. G. Prada Moroni ${ }^{23}$, E. Prandini ${ }^{4}$, I. Puljak ${ }^{5}$, J. R. Garcia ${ }^{7}$, I. Reichardt ${ }^{4}$, W. Rhode ${ }^{16}$, M. Ribó $^{20}$, J. Rico ${ }^{13}$, T. Saito ${ }^{21}$,
\end{abstract}

K. Satalecka ${ }^{12}$, S. Schroeder ${ }^{16}$, T. Schweizer ${ }^{7}$, S. N. Shore ${ }^{23}$, A. Sillanpää ${ }^{18}$, J. Sitarek ${ }^{11}$, I. Šnidarićc ${ }^{5}$, D. Sobczynska ${ }^{11}$, A. Stamerra ${ }^{3}$, M. Strzys $^{7}$,

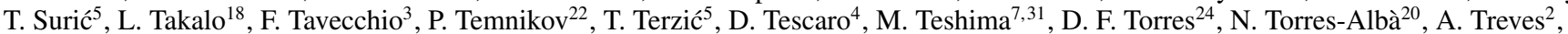

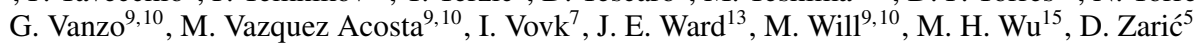

H.E.S.S. Collaboration: H. Abdalla ${ }^{35}$, A. Abramowski ${ }^{36}$, F. Aharonian ${ }^{37,38,39}$, F. Ait Benkhali ${ }^{37}$, A. G. Akhperjanian ${ }^{40,39}$, T. Andersson ${ }^{44}$,

E. O. Angüner ${ }^{55}$, M. Arakawa ${ }^{77}$, M. Arrieta ${ }^{49}$, P. Aubert ${ }^{58}$, M. Backes ${ }^{42}$, A. Balzer ${ }^{43}$, M. Barnard ${ }^{35}$, Y. Becherini ${ }^{44}$, J. Becker Tjus $^{55}$, D. Berge ${ }^{46}$,

S. Bernhard ${ }^{47}$, K. Bernlöhr ${ }^{37}$, R. Blackwell ${ }^{48}$, M. Böttcher ${ }^{35}$, C. Boisson ${ }^{49}$, J. Bolmont ${ }^{50}$, P. Bordas ${ }^{37, \star}$, J. Bregeon $^{51}$, F. Brun ${ }^{60, \star}$, P. Brun $^{52}$, M. Bryan ${ }^{43}$, M. Büchele ${ }^{70}$, T. Bulik ${ }^{53}$, M. Capasso ${ }^{63}$, J. Carr ${ }^{54}$, S. Casanova ${ }^{53,37}$, M. Cerruti ${ }^{50}$, N. Chakraborty ${ }^{37}$, R. Chalme-Calvet ${ }^{50}$,

R. C. G. Chaves ${ }^{51,56}$, A. Chen ${ }^{57}$, J. Chevalier ${ }^{58}$, M. Chrétien ${ }^{50}$, M. Coffaro ${ }^{63}$, S. Colafrancesco ${ }^{57}$, G. Cologna ${ }^{59}$, B. Condon ${ }^{60}$, J. Conrad ${ }^{61,62}$,

Y. Cui ${ }^{63}$, I. D. Davids ${ }^{35,42}$, J. Decock ${ }^{52}$, B. Degrange ${ }^{64}$, C. Deil ${ }^{37}$, J. Devin ${ }^{51}$, P. deWilt ${ }^{48}$, L. Dirson ${ }^{36}$, A. Djannati-Atai ${ }^{65}$, W. Domainko ${ }^{37}$,

A. Donath ${ }^{37}$, L. O'C. Drury ${ }^{38}$, K. Dutson ${ }^{67}$, J. Dyks ${ }^{68}$, T. Edwards ${ }^{37}$, K. Egberts ${ }^{69}$, P. Eger ${ }^{37}$, J.-P. Ernenwein ${ }^{54}$, S. Eschbach ${ }^{70}$, C. Farnier ${ }^{61,44}$,

S. Fegan ${ }^{64}$, M. V. Fernandes ${ }^{36}$, A. Fiasson ${ }^{58}$, G. Fontaine ${ }^{64}$, A. Förster ${ }^{37}$, S. Funk ${ }^{70}$, M. Füßling ${ }^{71}$, S. Gabici ${ }^{65}$, M. Gajdus ${ }^{41}$, Y. A. Gallant ${ }^{51}$,

T. Garrigoux ${ }^{35}$, G. Giavitto ${ }^{71}$, B. Giebels ${ }^{64}$, J. F. Glicenstein ${ }^{52}$, D. Gottschall ${ }^{63}$, A. Goyal ${ }^{72}$, M.-H. Grondin ${ }^{70}$, J. Hahn ${ }^{37}$, M. Haupt ${ }^{71}$, J. Hawkes ${ }^{48}$, G. Heinzelmann ${ }^{36}$, G. Henri ${ }^{66}$, G. Hermann ${ }^{37}$, O. Hervet ${ }^{49,79}$, J. A. Hinton ${ }^{37}$, W. Hofmann ${ }^{37}$, C. Hoischen ${ }^{69}$, M. Holler ${ }^{64}$, D. Horns ${ }^{36}$, A. Ivascenko ${ }^{35}$, H. Iwasaki $^{77}$, A. Jacholkowska ${ }^{50}$, M. Jamrozy ${ }^{72}$, M. Janiak ${ }^{68}$, D. Jankowsky ${ }^{70}$, F. Jankowsky ${ }^{59}$, M. Jingo $^{57}$, T. Jogler $^{70}$, L. Jouvin ${ }^{65}$, I. Jung-Richardt ${ }^{70}$, M. A. Kastendieck ${ }^{36}$, K. Katarzyński ${ }^{73}$, M. Katsuragawa ${ }^{78}$, U. Katz ${ }^{70}$, D. Kerszberg ${ }^{50}$, D. Khangulyan ${ }^{77}$ B. Khélifi ${ }^{65}$, M. Kieffer ${ }^{50}$, J. King ${ }^{37}$, S. Klepser ${ }^{71}$, D. Klochkov ${ }^{63}$, W. Kluźniak ${ }^{68}$, D. Kolitzus ${ }^{57}$, Nu. Komin ${ }^{57}$, K. Kosack ${ }^{52}$, S. Krakau ${ }^{45}$, M. Kraus ${ }^{70}$, P. P. Krüger ${ }^{35}$, H. Laffon ${ }^{60}$, G. Lamanna ${ }^{58}$, J. Lau ${ }^{48}$, J.-P. Lees ${ }^{58}$, J. Lefaucheur ${ }^{49}$, V. Lefranc ${ }^{52}$, A. Lemière ${ }^{65}$,

M. Lemoine-Goumard ${ }^{60}$, J.-P. Lenain ${ }^{50}$, E. Leser ${ }^{69}$, T. Lohse ${ }^{41}$, M. Lorentz ${ }^{52}$, R. Liu ${ }^{37}$, R. López-Coto ${ }^{37}$, I. Lypova ${ }^{71}$, V. Marandon ${ }^{37}$,

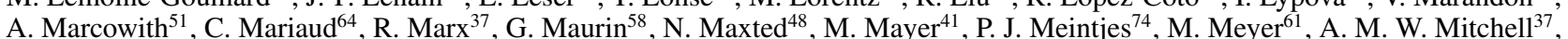

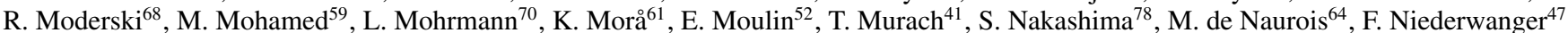

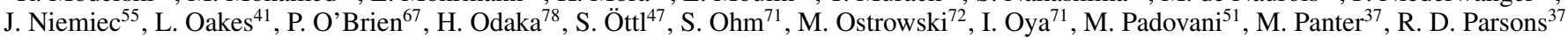

N. W. Pekeur ${ }^{35}$, G. Pelletier ${ }^{66}$, C. Perennes ${ }^{50}$, P.-O. Petrucci ${ }^{66}$, B. Peyaud ${ }^{52}$, Q. Piel ${ }^{58}$, S. Pita ${ }^{65}$, H. Poon ${ }^{37}$, D. Prokhorov ${ }^{44}$, H. Prokoph $^{44}$, G. Pühlhofer ${ }^{63}$, M. Punch ${ }^{65,44}$, A. Quirrenbach ${ }^{59}$, S. Raab ${ }^{70}$, A. Reimer ${ }^{47}$, O. Reimer ${ }^{47}$, M. Renaud ${ }^{51}$, R. de los Reyes ${ }^{37}$, S. Richter ${ }^{35}$, F. Rieger ${ }^{37,75}$, C. Romoli ${ }^{38}$, G. Rowell ${ }^{48}$, B. Rudak ${ }^{68}$, C. B. Rulten ${ }^{49}$, S. Safi-Harb ${ }^{80}$, V. Sahakian ${ }^{40,39}$, S. Saito ${ }^{77}$, D. Salek ${ }^{76}$, D. A. Sanchez ${ }^{58}$, A. Santangelo ${ }^{63}$, M. Sasaki ${ }^{63}$, R. Schlickeiser ${ }^{45}$, F. Schüssler ${ }^{52}$, A. Schulz ${ }^{71}$, U. Schwanke ${ }^{41}$, S. Schwemmer ${ }^{59}$, M. Seglar-Arroyo $^{52}$, M. Settimo $^{50}$, A. S. Seyffert ${ }^{35}$, N. Shafi ${ }^{57}$, I. Shilon ${ }^{70}$, R. Simoni ${ }^{43}$, H. Sol $^{49}$, F. Spanier ${ }^{35}$, G. Spengler ${ }^{61}$, F. Spies ${ }^{36}$, Ł. Stawarz $^{72}$, R. Steenkamp ${ }^{42}$, C. Stegmann ${ }^{69,71}$, K. Stycz ${ }^{71}$, I. Sushch ${ }^{35}$, T. Takahashi ${ }^{78}$, J.-P. Tavernet ${ }^{50}$, T. Tavernier ${ }^{65}$, A. M. Taylor ${ }^{38}$, R. Terrier ${ }^{65}$, L. Tibaldo $^{37}$, D. Tiziani ${ }^{70}$, M. Tluczykont ${ }^{36}$, C. Trichard ${ }^{54}$, N. Tsuji ${ }^{77}$, R. Tuffs ${ }^{37}$, Y. Uchiyama ${ }^{77}$, D. J. van der Walt ${ }^{35}$, C. van Eldik ${ }^{70}$, C. van Rensburg ${ }^{35}$, B. van Soelen ${ }^{74}$, G. Vasileiadis ${ }^{51}$, J. Veh ${ }^{70}$, C. Venter ${ }^{35}$, A. Viana ${ }^{37}$, P. Vincent ${ }^{50}$, J. Vink ${ }^{43}$, F. Voisin ${ }^{48}$, H. J. Völk ${ }^{37}$, T. Vuillaume ${ }^{58}$, Z. Wadiasingh ${ }^{35}$, S. J. Wagner ${ }^{59}$, P. Wagner ${ }^{41}$, R. M. Wagner ${ }^{61}$, R. White ${ }^{37}$, A. Wierzcholska ${ }^{55}$, P. Willmann ${ }^{70}$, A. Wörnlein ${ }^{70}$, D. Wouters ${ }^{52}$, R. Yang ${ }^{37}$, V. Zabalza ${ }^{67}$, D. Zaborov ${ }^{64}$, M. Zacharias ${ }^{59}$, R. Zanin ${ }^{37}$, A. A. Zdziarski ${ }^{68}$, A. Zech ${ }^{49}$, F. Zefi ${ }^{64}$, A. Ziegler ${ }^{70}$, and N. Żywucka ${ }^{72}$

(Affiliations can be found after the references)

Received 14 May 2017 / Accepted 29 June 2017

\section{ABSTRACT}

Context. The large jet kinetic power and non-thermal processes occurring in the microquasar SS 433 make this source a good candidate for a very high-energy (VHE) gamma-ray emitter. Gamma-ray fluxes above the sensitivity limits of current Cherenkov telescopes have been predicted for both the central X-ray binary system and the interaction regions of SS 433 jets with the surrounding W50 nebula. Non-thermal emission at lower energies has been previously reported, indicating that efficient particle acceleration is taking place in the system.

Aims. We explore the capability of SS 433 to emit VHE gamma rays during periods in which the expected flux attenuation due to periodic eclipses $\left(P_{\mathrm{orb}} \sim 13.1\right.$ days $)$ and precession of the circumstellar disk $\left(P_{\mathrm{pre}} \sim 162\right.$ days $)$ periodically covering the central binary system is expected to be at 
A\&A 612, A14 (2018)

its minimum. The eastern and western SS 433/W50 interaction regions are also examined using the whole data set available. We aim to constrain some theoretical models previously developed for this system with our observations.

Methods. We made use of dedicated observations from the Major Atmospheric Gamma Imaging Cherenkov telescopes (MAGIC) and High Energy Spectroscopic System (H.E.S.S.) of SS 433 taken from 2006 to 2011. These observation were combined for the first time and accounted for a total effective observation time of $16.5 \mathrm{~h}$, which were scheduled considering the expected phases of minimum absorption of the putative VHE emission. Gamma-ray attenuation does not affect the jet/medium interaction regions. In this case, the analysis of a larger data set amounting to $\sim 40-80 \mathrm{~h}$, depending on the region, was employed.

Results. No evidence of VHE gamma-ray emission either from the central binary system or from the eastern/western interaction regions was found. Upper limits were computed for the combined data set. Differential fluxes from the central system are found to be $\lesssim 10^{-12}-10^{-13} \mathrm{TeV}^{-1} \mathrm{~cm}^{-2} \mathrm{~s}^{-1}$ in an energy interval ranging from $\sim$ few $\times 100 \mathrm{GeV}$ to $\sim$ few TeV. Integral flux limits down to $\sim 10^{-12}-10^{-13} \mathrm{ph} \mathrm{cm}^{-2} \mathrm{~s}^{-1}$ and $\sim 10^{-13}-10^{-14} \mathrm{ph} \mathrm{cm}{ }^{-2} \mathrm{~s}^{-1}$ are obtained at 300 and $800 \mathrm{GeV}$, respectively. Our results are used to place constraints on the particle acceleration fraction at the inner jet regions and on the physics of the jet/medium interactions.

Conclusions. Our findings suggest that the fraction of the jet kinetic power that is transferred to relativistic protons must be relatively small in SS $433, q_{\mathrm{p}} \leq 2.5 \times 10^{-5}$, to explain the lack of TeV and neutrino emission from the central system. At the SS $433 /$ W50 interface, the presence of magnetic fields $\gtrsim 10 \mu \mathrm{G}$ is derived assuming a synchrotron origin for the observed X-ray emission. This also implies the presence of high-energy electrons with $E_{\mathrm{e}^{-}}$up to $50 \mathrm{TeV}$, preventing an efficient production of gamma-ray fluxes in these interaction regions.

Key words. gamma rays: general - stars: black holes - X-rays: binaries - ISM: jets and outflows

\section{The SS $433 /$ W50 system}

SS 433 (RA $19^{\mathrm{h}} 11^{\mathrm{m}} 49.57^{\mathrm{s}}$, Dec $4^{\circ} 58^{\prime \prime} 57.9^{\prime}$ ) is the first binary system containing a stellar-mass compact object in which relativistic jets were discovered (Abell \& Margon 1979; Fabian \& Rees 1979). Located at a distance of $5.5 \pm 0.2 \mathrm{kpc}$ (Blundell \& Bowler 2004; Lockman et al. 2007), SS 433 is an eclipsing X-ray binary system containing a black hole that is most likely $\sim 10-20 M_{\odot}$ (Margon 1984) orbiting a $\sim 30 M_{\odot}$ A3-7 supergiant star in a circular orbit with radius $79-86 R_{\odot}$ (Fabrika 2004). SS 433 is extremely bright with a bolometric luminosity of $L_{\mathrm{bol}} \sim 10^{40} \mathrm{erg} \mathrm{s}^{-1}$ (Cherepashchuk 2002) peaking at ultraviolet wavelengths. The source displays the most powerful jets known in our Galaxy, with $L_{\text {jet }} \gtrsim 10^{39} \mathrm{erg} \mathrm{s}^{-1}$ (Dubner et al. 1998; Margon 1984; Marshall et al. 2002), ejected at a relativistic velocity of $0.26 c$ (Margon \& Anderson 1989). The jets show a precessional period of $\sim 162.4$ days with a half opening angle of $\theta_{\text {pre }} \approx 21^{\circ}$ with respect to the normal to the orbital plane, with precessional phase $\Psi_{\text {pre }}=0$ defined as the phase with the maximum exposure of the accretion disk to the observer (Fabrika 1993). The inclination of the jets with respect to the line of sight subtends an angle of $i \approx 78^{\circ}$ (Eikenberry et al. 2001). This value is, however, time dependent owing to precession.

Both the high luminosity of SS 433 and its enormous jet power are thought to be a consequence of the persistent regime of supercritical accretion onto the compact object via Roche lobe overflow at a rate of $\dot{M} \sim 10^{-4} M_{\odot} \mathrm{yr}^{-1}$. In addition, SS 433 is one of the only two X-ray binary systems in which the presence of baryons in their jets has been found (Kotani et al. 1994); the other system is 4U 1630-47 (Díaz Trigo et al. 2013). Clouds of plasma with baryonic content propagate along ballistic trajectories to large distances without appreciable deceleration. At growing distances from the source, the collimated jets (with opening angle of $\approx 1.2^{\circ}$ ) can be distinguished in the X-ray, optical, and radio bands. The X-ray jets give rise to lines of highly ionized heavy elements (Kotani et al. 1994; Marshall et al. 2002). The emission is produced by hot gas $\left(T \sim 10^{8} \mathrm{~K}\right)$, which cools due to expansion and radiative losses while propagating outwards. Plasma at $T \sim 10^{7} \mathrm{~K}$ is, however, still observed at large distances along the jet, indicating that a continuous source of heating is required to maintain the observed emission (Migliari et al. 2002). At radio wavelengths, the observed synchrotron flux density is about $1 \mathrm{Jy}$ at $1 \mathrm{GHz}$ with luminosities reaching $\sim 4 \times 10^{32} \mathrm{erg} \mathrm{s}^{-1}$.

\footnotetext{
* Corresponding authors: H.E.S.S. Collaboration, e-mail: contact.hess@hess-experiment.eu; MAGIC Collaboration, e-mail: aloramas@iac.es; pere.munar@iaps.inaf.it
}

SS 433 is surrounded by the radio shell of W50, which is a large $2^{\circ} \times 1^{\circ}$ nebula catalogued as SNR G39.7-2.0 (Green 2006). Its present morphology is thought to be the result of the interaction between the jets of SS 433 and the surrounding medium (Goodall et al. 2011). This scenario is supported by the position of SS 433 at the centre of W50, the elongation of the nebula in the east-west direction along the axis of precession of the jets (forming the so-called "ears" of W50; see Safi-Harb \& Ögelman 1997), the presence of radio, IR, optical and X-ray emitting regions also aligned with the jet precession axis, and the structure of the magnetic field through the observation of linearly polarized radio emission in the SS 433/W50 region (Farnes et al. 2017). At a distance of $\sim 10^{20} \mathrm{~cm}$, or about $\sim 30 \mathrm{pc}$, the outflowing jets are decelerated and, together with an enhanced intensity of the emission at radio wavelengths, large-scale X-ray lobes are observed. The extended X-ray emission is mostly of non-thermal origin and generally much softer than the emission from the central source (hard X-ray emission is found however in the eastern interaction regions; see Safi-Harb \& Ögelman 1997). At the position of the maximum of the X-ray extended emission, optical filaments perpendicular to the jet precession axis are found. Spectral analysis shows that these filaments are formed by the sweeping up of the interstellar gas and display a proper motion of $50-90 \mathrm{~km} \mathrm{~s}^{-1}$ (Zealey et al. 1980; Fabrika 2004). At larger distances, beyond the W50 length scales, the presence of molecular clouds aligned in the direction of SS 433 jets has been reported (Yamamoto et al. 2008). These clouds, which extend for $\sim 250 \mathrm{pc}$ at a distance of $5 \mathrm{kpc}$, may have formed through the interaction of SS 433 jets with the interstellar H I gas, which would imply that SS 433 jets are more extended by a factor of $\sim 3$ than the observed X-ray jets (Yamamoto et al. 2008).

\section{Gamma-ray emission and absorption processes in SS 433/W50}

SS 433 is an exceptional laboratory to test theoretical predictions of high $(100 \mathrm{MeV}<E<100 \mathrm{GeV})$ and very high-energy (VHE; $E>100 \mathrm{GeV}$ ) emission produced in microquasar jets (see e.g. Levinson \& Blandford 1996; Atoyan \& Aharonian 1999; Kaufman Bernadó et al. 2002; Bosch-Ramon et al. 2006; Orellana et al. 2007; Reynoso et al. 2008b; Bosch-Ramon \& Khangulyan 2009). In a leptonic framework, gamma rays could be produced through inverse Compton (IC) scattering of ambient photon fields, which are dominated in this case by the supergiant companion star and the UV and 
mid-IR emission from the extended accretion disk (Gies et al. 2002; Fuchs et al. 2006). In addition, synchrotron-self Compton emission and the interaction of accelerated electrons with jet ions through relativistic Bremsstrahlung processes could also generate VHE fluxes (Aharonian \& Atoyan 1998). In a hadronic scenario, interactions of relativistic protons in the jet produce gamma rays through $\pi^{0}$ decay (see e.g. Reynoso et al. 2008b, for a detailed study applied to SS 433). The target ions could be provided both by the companion and disk winds or by the pool of thermal protons outflowing within the jets.

Gamma rays, when produced in the inner regions of SS 433, can be strongly attenuated (see e.g. Reynoso et al. 2008a). Both the donor star and compact object are thought to be embedded in a thick extended envelope (Zwitter et al. 1991), which forms as a result of the supercritical accretion rate onto the compact object, and provides a dense low-energy UV and mid-IR radiation field in which VHE photons are absorbed. In addition, about $10^{-4} M_{\odot} \mathrm{yr}^{-1}$ are expelled within the $\sim 30^{\circ}$ half opening angle subtended by the envelope (Fabrika \& Sholukhova 2008). Therefore, absorption of VHE gamma rays can also occur due to gamma-nucleon interactions through photo-pion and photo-pair production processes, whereas the photon field of the companion can also effectively reduce the gamma-ray flux through pair creation (Reynoso et al. 2008a). Such strong absorption is expected to occur along $\sim 80 \%$ of the SS 433 precession cycle with a maximum at precession phase $\Psi_{\text {pre }} \approx 0.5$, and during the regular eclipses of the compact object by the donor star at orbital phases $\phi_{\text {orb }} \approx 0$.

The interaction regions between the SS 433 jets and the surrounding W50 nebula could also produce VHE gamma-ray emission, for example through IC scattering of cosmic microwave background (CMB) photons by electrons accelerated at the eastern $(e 1, e 2, e 3)$ and western $(w 1, w 2)$ termination regions (Safi-Harb \& Ögelman 1997; Aharonian \& Atoyan 1998; Bordas et al. 2009), or through pp interactions if protons are efficiently accelerated at the interaction shocks (Heinz \& Sunyaev 2002; Bosch-Ramon et al. 2005). Non-thermal emission from these regions has indeed been observed from radio to $\mathrm{X}$-ray energies (Brinkmann et al. 1996, 2007; Dubner et al. 1998; Safi-Harb \& Ögelman 1997; Safi-Harb \& Petre 1999; Fuchs 2002).

Recently, high-energy gamma-ray emission from a source associated with SS 433/W50 has been reported from the analysis of $\sim 5$ yr of Fermi-LAT archival data (Bordas et al. 2015). The relatively large point spread function (PSF) of the FermiLAT at the sub-GeV energies in which the source is detected, larger than $\sim 1.5^{\circ}$, prevents from an accurate localization of the emitter. At VHEs, SS 433/W50 remains so far undetected. Upper limits have been reported by the CANGAROO-II and HEGRA Collaborations, at the level of $2-3 \%$ of the Crab nebula flux above $800 \mathrm{GeV}$ (Hayashi et al. 2009; Aharonian et al. 2005), following extended observation campaigns including both the central system and the jet/medium interaction regions. The selected dates of these observations, however, did not account for the gamma-ray absorption affecting the inner system. Such constraints were instead accounted for in observations of SS 433 performed by VERITAS in 2007 and MAGIC in 2008, for which upper limits are reported in Saito et al. (2009) and Guenette \& for the VERITAS Collaboration (2009), respectively.

In this work, a search for VHE emission from the microquasar SS 433 with the Major Atmospheric Gamma Imaging Cherenkov telescopes (MAGIC) and High Energy Spectroscopic System (H.E.S.S.) Imaging Atmospheric Cherenkov Telescopes
(IACTs) is reported, following dedicated observations of the source spanning several years and taken at orbital/precession phases where gamma-ray absorption should be minimal. The SS 433/W50 interaction regions are also investigated, for which a wider data set is used that is not restricted to the low-absorption phases criterion applied to the study of the inner system. The observations and analysis results are described in Sect. 3 and are later discussed in Sect. 4.

\section{VHE observations, analysis, and results}

\subsection{H.E.S.S. and MAGIC observations}

Observations of SS 433/W50 were conducted with the H.E.S.S. and MAGIC Cherenkov telescope arrays. The H.E.S.S. is an IACT array located in the Khomas highland of Namibia $\left(23^{\circ} \mathrm{S}\right.$, $16^{\circ} \mathrm{E}, 1800 \mathrm{~m}$ above the sea level). In its first phase, the system consisted of four identical $13 \mathrm{~m}$ diameter imaging Cherenkov telescopes, covering a field of view (FoV) of about $5^{\circ}$ diameter (Bernlöhr et al. 2003). A major upgrade took place in 2012 with the addition of CT-5, which is a $28 \mathrm{~m}$ diameter telescope at the centre of the array. The data presented in this paper makes use of observations taken during the H.E.S.S.-I phase only (CT1-4 configuration). In this configuration, H.E.S.S. is able to detect at a $5 \sigma$ statistical significance level a source with $\sim 0.7 \%$ of the Crab nebula flux in $50 \mathrm{~h}$ of observations (Aharonian et al. 2006a). The MAGIC is a stereoscopic system of two $17 \mathrm{~m}$ diameter Cherenkov telescopes located at the observatory El Roque de Los Muchachos $\left(28^{\circ} \mathrm{N}, 18^{\circ} \mathrm{W}, 2200 \mathrm{~m}\right.$ above the sea level) on La Palma, Canary Islands, Spain. Each telescope is composed of a pixelized camera with a FoV of $3.5^{\circ}$. The sensitivity of MAGIC at the time of the observations was $\sim 0.76 \% \pm 0.03 \%$ of the Crab nebula flux in $50 \mathrm{~h}$ above $290 \mathrm{GeV}$ (Aleksić et al. 2012a).

The H.E.S.S. and MAGIC data set includes observations of SS 433/W50 taken in 2006, 2007, 2009, 2010, and 2011. Whereas the 2006 and 2007 H.E.S.S. observations were part of the H.E.S.S. Galactic Plane Survey (HGPS; H.E.S.S. Collaboration 2018b), both the MAGIC and H.E.S.S. campaigns in 2009, 2010, and 2011 were dedicated to SS 433. Making use of the ephemeris provided by Goranskij (2011), the latter were scheduled at times in which the source was found at orbital and precession phases $\Psi$ where absorption of its putative VHE emission is expected to be at its minimum, $\Psi_{\text {pre }}=0.9$ 0.1 (Reynoso et al. 2008a). The total H.E.S.S. effective exposure time on SS 433/W50 amounts to $45 \mathrm{~h}$ of data after standard quality selection cuts. A total effective exposure time of $8.7 \mathrm{~h}$ is available for the central system after selecting low-absorption precession/orbital phases. In May and June 2010, MAGIC performed observations of SS 433 in stereo mode for $10 \mathrm{~h}$. After quality cuts, $7.8 \mathrm{~h}$ of good data remained. A summary of the H.E.S.S. and MAGIC observations of SS 433 is collected in Table 1.

The H.E.S.S. observations of the central system were performed at zenith angles ranging between $28^{\circ}$ to $54^{\circ}$, with an average of $34^{\circ}$, while MAGIC observed at zenith angles between $24^{\circ}$ and $30^{\circ}$. The observations in both H.E.S.S and MAGIC were performed in wobble mode (Fomin et al. 1994), with an offset of $0.4^{\circ}$ for MAGIC and $0.7^{\circ}$ for H.E.S.S., respectively, away from the source position to simultaneously evaluate the background. This observation mode allowed imaging of not only the central binary system, but also the eastern $(e 1, e 2, e 3)$ and western $(w 1, w 2)$ interaction regions with the W50 nebula. In the case of H.E.S.S., the interaction regions were also observed as part of the HGPS programme (Aharonian et al. 2006b; H.E.S.S. Collaboration 2018b), at zenith angles between $25^{\circ}$ to 
Table 1. Observations of SS 433 performed by H.E.S.S. and MAGIC telescopes.

\begin{tabular}{ccccc}
\hline \hline Instrument & Epoch & $\begin{array}{c}\text { Zenith angle } \\
{\left[{ }^{\circ}\right]}\end{array}$ & $\begin{array}{c}\text { Time } \\
{[\mathrm{h}]}\end{array}$ & $\Psi_{\text {pre }}$ \\
\hline H.E.S.S. & 30 May-5 June 2006 & $28-44$ & 3.0 & $0.95-0.99$ \\
& 30 September-12 October 2007 & $38-46$ & 3.1 & $0.96-0.04$ \\
& 3-17 July 2009 & $33-54$ & 0.9 & $0.92-0.01$ \\
& 9-10 May 2011 & $28-37$ & 2.1 & $0.07-0.08$ \\
\hline MAGIC & 20-23 May 2010 & $24-29$ & 5.6 & $0.90-0.92$ \\
& 08-10 June 2010 & $24-30$ & 4.4 & $0.01-0.03$ \\
\hline
\end{tabular}

Notes. The table includes the date of the observations, telescope configuration or number of operating telescopes, zenith angle range, observation live-time, and corresponding precessional phase (based on ephemeris by Goranskij 2011).

Table 2. Integral H.E.S.S. and MAGIC flux upper limits derived for SS 433 during low-absorption orbital/precessional phases and for the eastern/western interaction regions indicated in Fig. 1 using all available data.

\begin{tabular}{|c|c|c|c|c|}
\hline Region & IACT & $\begin{array}{l}t_{\text {eff }} \\
{[\mathrm{h}]} \\
\end{array}$ & $\begin{array}{c}300 \mathrm{GeV} \text { UL } \\
{\left[\mathrm{cm}^{-2} \mathrm{~s}^{-1}\right]} \\
\end{array}$ & $\begin{array}{c}800 \mathrm{GeV} \mathrm{UL} \\
{\left[\mathrm{cm}^{-2} \mathrm{~s}^{-1}\right]}\end{array}$ \\
\hline SS 433 & HEGRA & 96.3 & - & $8.9 \times 10^{-13}$ \\
\hline $\mathrm{RA}=19^{\mathrm{h}} 11^{\mathrm{m}} 50^{\mathrm{s}}$ & H.E.S.S. & 8.7 & $2.3 \times 10^{-12}$ & $3.9 \times 10^{-13}$ \\
\hline Dec $=04^{\circ} 58^{\prime} 58^{\prime \prime}$ & MAGIC & 7.8 & $1.8 \times 10^{-12}$ & $4.3 \times 10^{-13}$ \\
\hline \multirow{3}{*}{$\begin{array}{c}e 1 \\
\mathrm{RA}=19^{\mathrm{h}} 13^{\mathrm{m}} 37^{\mathrm{s}} \\
\operatorname{Dec}=04^{\circ} 55^{\prime} 48^{\prime \prime} \\
\left(r=0.05^{\circ}\right)\end{array}$} & HEGRA & 72.0 & - & $6.2 \times 10^{-13}$ \\
\hline & H.E.S.S. & 36.5 & $6.8 \times 10^{-13}$ & $1.4 \times 10^{-13}$ \\
\hline & MAGIC & 7.8 & $1.6 \times 10^{-11}$ & $1.9 \times 10^{-12}$ \\
\hline \multirow{3}{*}{$\begin{array}{c}e 2 \\
\mathrm{RA}=19^{\mathrm{h}} 14^{\mathrm{m}} 20^{\mathrm{s}} \\
\operatorname{Dec}=04^{\circ} 54^{\prime} 25^{\prime \prime} \\
\left(r=0.17^{\circ}\right)\end{array}$} & HEGRA & 73.1 & - & $9.2 \times 10^{-13}$ \\
\hline & H.E.S.S. & 34.8 & $6.0 \times 10^{-13}$ & $1.3 \times 10^{-13}$ \\
\hline & MAGIC & 7.8 & $1.7 \times 10^{-11}$ & $2.0 \times 10^{-12}$ \\
\hline \multirow{3}{*}{$\begin{array}{c}e 3 \\
\mathrm{RA}=19^{\mathrm{h}} 16^{\mathrm{m}} 04^{\mathrm{s}} \\
\operatorname{Dec}=04^{\circ} 50^{\prime} 13^{\prime \prime} \\
\left(r=0.25^{\circ}\right)\end{array}$} & HEGRA & 68.8 & - & $9.0 \times 10^{-13}$ \\
\hline & H.E.S.S. & 18.9 & $1.1 \times 10^{-12}$ & $9.3 \times 10^{-13}$ \\
\hline & MAGIC & 7.8 & $8.7 \times 10^{-12}$ & $6.1 \times 10^{-13}$ \\
\hline \multirow{3}{*}{$\begin{array}{c}w 1 \\
\mathrm{RA}=19^{\mathrm{h}} 10^{\mathrm{m}} 37^{\mathrm{s}} \\
\operatorname{Dec}=05^{\circ} 02^{\prime} 13^{\prime \prime} \\
\left(r=0.07^{\circ}\right)\end{array}$} & HEGRA & 104.9 & - & $6.7 \times 10^{-13}$ \\
\hline & H.E.S.S. & 62.5 & $2.2 \times 10^{-13}$ & $4.0 \times 10^{-14}$ \\
\hline & MAGIC & 7.8 & $1.3 \times 10^{-11}$ & $2.2 \times 10^{-12}$ \\
\hline \multirow{3}{*}{$\begin{array}{c}w 2 \\
\mathrm{RA}=19^{\mathrm{h}} 09^{\mathrm{m}} 40^{\mathrm{s}} \\
\operatorname{Dec}=05^{\circ} 02^{\prime} 13^{\prime \prime} \\
\left(r=0.07^{\circ}\right)\end{array}$} & HEGRA & 100.7 & - & $9.0 \times 10^{-13}$ \\
\hline & H.E.S.S. & 60.8 & $3.2 \times 10^{-13}$ & $7.6 \times 10^{-14}$ \\
\hline & MAGIC & 7.8 & $1.4 \times 10^{-11}$ & $2.6 \times 10^{-12}$ \\
\hline
\end{tabular}

Notes. The results obtained with HEGRA (Aharonian et al. 2005) are also included for comparison. Columns denote from left to right: the region of study (with coordinates and extension radius for the interaction regions) IACT instrument, effective exposure time, energy threshold for the UL calculation, and integral flux UL computed at 99\% C.L.

$57^{\circ}$, with an average of $35^{\circ}-38^{\circ}$ depending on the region. The total exposure time varies from region to region (see Table 2).

\subsection{Analysis}

Data analysis was performed following the standard analysis procedure for each of the two instruments (see Aharonian et al. 2006a, for H.E.S.S.; and Aleksić et al. 2016, for MAGIC analysis details). The imaging technique is based on the parameterization of the images formed in the camera plane in order to extract the information contained in the shower with the Hillas parameters (Hillas 1985). The signal extraction was performed by the reconstruction and calibration of the size and arrival time of the Cherenkov pulses. The event reconstruction was obtained by image cleaning and shower parameterization, whereas the signal and background discrimination and energy estimation were obtained by comparison of the Hillas parameters with look-up tables for a given shower intensity and impact distance (see Aharonian et al. 2006a; Aleksić et al. 2012a), or by training an algorithm to perform gamma/hadron separation via the random forest (RF) method (Albert et al. 2008). The event direction was derived in stereoscopic observations from the intersection of the major axes of the shower images in multiple cameras. Finally, the signal was extracted geometrically from the angular distance $\theta^{2}$; i.e. the angular distance between the source position and the estimated source position for a given event. The signal is then determined by an upper cut in these angles, since gamma rays are reconstructed with small angles and the background follows a featureless, almost-flat distribution. For the H.E.S.S. analysis, an independent cross-check with the model analysis technique (de Naurois \& Rolland 2009) was performed, making use of an independent calibration procedure of the raw data, with both the analysis chains providing compatible results. Standard cuts were used, where a cut of 60 photoelectrons on the intensity of the extensive air showers is applied, providing a mean energy threshold of $\sim 287 \mathrm{GeV}$ for the analysis reported here. The energy threshold reached by MAGIC is $150 \mathrm{GeV}$. A point-like source was assumed for the analysis of SS 433. The interaction regions display extended emission at lower energies. To account for such extension, the MAGIC and H.E.S.S. analyses were optimized assuming a source radius ( $\theta$-cut) of $0.05^{\circ}, 0.17^{\circ}, 0.25^{\circ}, 0.07^{\circ}$, and $0.07^{\circ}$ for $e 1, e 2, e 3$, $w 1$, and $w 2$, respectively, derived from the extension of these regions observed at X-ray energies (see e.g. Safi-Harb \& Ögelman 1997; Safi-Harb \& Petre 1999; Aharonian et al. 2005, and references therein).

\subsection{Results}

The H.E.S.S. and MAGIC observations reported here do not show any significant VHE emission either for the central source SS 433 or for any of the interaction regions with the W50 nebula $e 1, e 2, e 3, w 1$, and $w 2$ (see Fig. 1). Integral upper limits Rolke et al. (2005) have been calculated at $E \geq 300 \mathrm{GeV}$ and at $E \geq 800 \mathrm{GeV}$; the latter allows for a comparison with previous results on the source reported by the HEGRA (Aharonian et al. 2005) Collaboration. The results are summarised in Table 2. A day-by-day analysis of the H.E.S.S. and MAGIC data sets was also performed without any signature of significant emission, which could suggest a flaring episode during the dates of observation.

The H.E.S.S. and MAGIC observations were used to compute the differential flux upper limits for the VHE emission from the central binary system at orbital/precession phases where absorption should be at its minimum. These limits were computed through a maximum-likelihood ratio test applied to 


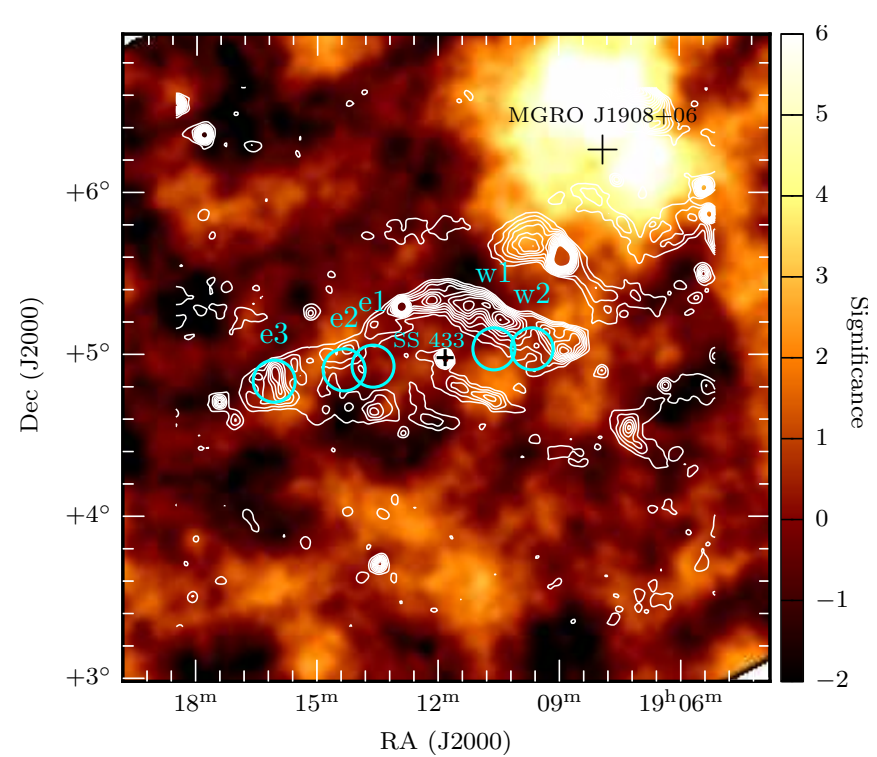

Fig. 1. Significance map, derived from the H.E.S.S. data, for the FoV centred at the position of SS 433/W50 at $E \geq 287 \mathrm{GeV}$. GB6 $4.85 \mathrm{GHz}$ radio contours (white, from Gregory et al. 1996) are superimposed. Cyan circles indicate the positions of the interaction regions $e 1, e 2, e 3$ (eastern "ear") and $w 1, w 2$ (western "ear"). The bright source located north-west of SS 433 is MGRO J1908+06 (Abdo et al. 2007).

the combined data sets obtained by both observatories. Events in the signal region $\left(n_{\mathrm{ON}}\right)$ and in the background control regions $\left(n_{\mathrm{OFF}}\right)$ from each instrument are collected in addition to the ratio of the areas in the signal and the background regions $(\alpha)$, effective area $\left(A_{\text {eff }}\right)$ and effective observing time $t_{\text {eff }}$ corresponding to the observations of each instrument. A likelihood profile is then computed in each studied energy bin $\left(\Delta E_{\mathrm{i}}\right)$ for both the signal and background distributions. Systematic uncertainties are accounted for through the inclusion of additional likelihood profiles for the distributions of $\alpha, A_{\text {eff }}$, and energy resolution, assuming systematics at the level of $\delta \alpha=10 \%, \delta A_{\text {eff }}=15 \%$, and $\delta E_{\mathrm{i}}=15 \%$ for the measurements of these quantities by each instrument (Aharonian et al. 2006a; Aleksić et al. 2016). The inclusion of these systematics results in an enhancement by $\sim 15 \%$ to $30 \%$ on the final combined differential flux upper limit values, depending on the studied energy bin. To obtain the final combined differential flux upper limits, a likelihood ratio test is employed assuming a given range of values for the normalization factor of the gamma-ray differential spectrum, $N_{0}$. From the maximum of the likelihood profile, a $95 \%$ confidence interval for the differential upper limit in each energy bin $\Delta E_{\mathrm{i}}$ is derived through $\mathrm{d} N / \mathrm{d} E=N_{0} \times E^{-\Gamma}$, where a fixed spectral in$\operatorname{dex} \Gamma=2.7$ was assumed. The final differential upper limits are shown in Fig. 2, both for each instrument and the combined values, together with the Crab nebula flux, for reference, and the theoretical predictions on the gamma-ray flux from SS433 expected at low-absorption precession phases $\Psi \in[0.9,0.1]$ by Reynoso et al. (2008b).

\section{Discussion}

The H.E.S.S. and MAGIC observations reported here do not show any significant signal of VHE emission from SS 433/W50. The variable absorption of a putative VHE gamma-ray flux emitted from the inner regions of the binary system, which could be responsible for this non-detection, is accounted for in this study by selecting observations corresponding to precession/orbital phases where this absorption should be at its minimum. The

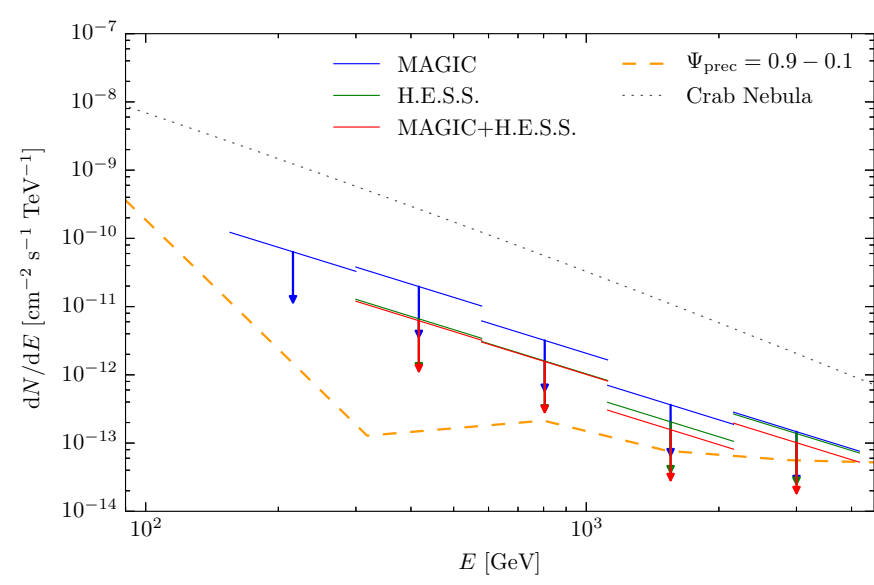

Fig. 2. Differential flux upper limits (95\% C.L.) from SS 433 obtained with MAGIC (blue), H.E.S.S. (green) and a combination of both telescopes (red) assuming a power-law with a spectral index $\Gamma=2.7$ for the differential gamma-ray flux. The predicted differential gamma-ray flux from Reynoso et al. (2008b) for precessional phases $\Psi_{\text {pre }} \in[0.9,0.1]$ in which absorption of VHE emission should be at its lower level is also displayed (dashed orange), together with the Crab Nebula flux, for reference (from Aleksić et al. 2012b).

combination of the MAGIC and H.E.S.S. observations in addition provides a relatively wide coverage of the relevant precession phases from 2006 to 2011. If a long-term super-orbital variability exists in SS 433 with timescales of $\sim$ few years, for example related to a varying jet injection power or the changing conditions of the absorber in the surroundings of the central compact object, such variability does not result in an enhancement of the TeV flux up to the detection level of current IACTs.

While SS 433 remains undetected at VHE, the system displays non-thermal emission at lower energies along the jets and/or at the SS 433/W50 interaction regions, which ensures the presence of an emitting population of relativistic particles in the system. In particular for the eastern nebula interaction sites, the observed synchrotron X-ray emission implies the presence of for example up to multi-TeV electrons in these regions (Safi-Harb \& Petre 1999).

By considering in detail the photon and matter fields both from the companion star and accretion/circumstellar disks, gamma-ray fluxes from SS 433/W50 have been predicted at a level of $\sim 10^{-12}-10^{-13} \mathrm{ph} \mathrm{cm}^{-2} \mathrm{~s}^{-1}$ (see e.g. Band \& Grindlay 1986; Aharonian \& Atoyan 1998; Reynoso et al. 2008b). Reynoso et al. (2008b) consider in particular $p p$ interactions between relativistic and cold protons in SS 433 jets during low-absorption precession/orbital phases, producing gamma-ray fluxes at $E_{\gamma} \geq 800 \mathrm{GeV}$ during these precession phases at a level of $\Phi_{\mathrm{VHE}} \approx 2.1 \times 10^{-12} \mathrm{ph} \mathrm{cm}^{-2} \mathrm{~s}^{-1}$. The general framework used to derive the relativistic proton distribution in Reynoso et al. (2008b) has been revised by Torres \& Reimer (2011), who report significant deviations of these proton fluxes for jets displaying large Lorentz factors and/or small viewing angles, for example blazar jets and gamma-ray bursts. In SS 433, with a moderate jet Lorentz factor of $1.036(v=0.26 c$; Abell \& Margon 1979) and a relatively large jet viewing angle, $\sim 78^{\circ}$ (Eikenberry et al. 2001), the correction factor on the fluxes predicted by Reynoso et al. (2008b) could be affected at the level of $\sim 20 \%$. The gamma-ray flux predicted by Reynoso et al. (2008) depends on the efficiency in transferring jet kinetic energy to the relativistic proton population, $q_{\mathrm{p}}$, which is treated in their model as a free parameter. Using the HEGRA upper limits to the VHE gamma-ray flux from SS $433, q_{\mathrm{p}}$ is constrained to 
be $\leq 2.9 \times 10^{-4}$. This upper limit is obtained however under the assumption that the HEGRA observations took place during a complete precessional cycle. With the H.E.S.S. and MAGIC upper limits reported here, a more stringent constraint on the fraction of power carried by relativistic protons in the SS 433 jets is obtained, $q_{\mathrm{p}} \lesssim 2.5 \times 10^{-5}$.

In a hadronic scenario, gamma rays from $\pi^{0}$-decay should also be accompanied by neutrinos from the decay of charged pions. The IceCube Collaboration analyzed the data around the position of SS 433/W50 with no significant detection of the source. An upper limit at $90 \%$ confidence level (C.L.) on the muon neutrino flux at $1 \mathrm{TeV}$ is set at $\phi_{\nu_{\mu}+\bar{v}_{\mu}}=0.65 \times$ $10^{-12} \mathrm{TeV} \mathrm{cm}^{-2} \mathrm{~s}^{-1}$ (Aartsen et al. 2014), using four years of data and assuming an $E^{-2}$ flux distribution. The model of Reynoso et al. (2008b) also predicts the neutrino flux emitted by this system with a neutrino differential flux at $1 \mathrm{TeV}$ of $\phi_{v}=2 \times$ $10^{-12} \mathrm{~cm}^{-2} \mathrm{~s}^{-1}$ averaged over all precessional phases. The IceCube upper limits can be used to put a limit on $q_{\mathrm{p}}$ of $\sim 3.3 \times 10^{-5}$, which is marginally less restrictive than the value obtained with the gamma-ray observations. However, the gamma-ray and neutrino flux estimates of Reynoso et al. (2008b) are based on a proton acceleration efficiency of $\eta=t_{\mathrm{acc}} / t_{\mathrm{gyr}} \sim 0.07 ; t_{\mathrm{acc}}$ is the acceleration time and $t_{\mathrm{gyr}}=c e B / E_{\mathrm{p}}$, where $B$ is the magnetic field in the accelerator region and $E_{\mathrm{p}}$ is the proton energy. Accounting for adiabatic and radiation losses, these authors derive a maximum energy for relativistic protons of $E_{\mathrm{p}} \leq 10^{3} \mathrm{TeV}$. Different values for the magnetic field, target proton densities, and/or adiabatic expansion velocities in the acceleration region would also imply variations in predicted gamma-ray and neutrino fluxes.

At the interaction regions of the jets of SS 433 with the surrounding W50 nebula, the X-ray spectra from the extended lobes are well represented by a power-law model (Moldowan \& Safi-Harb 2005); a synchrotron origin for this emission has been suggested (Safi-Harb \& Ögelman 1997; see also Safi-Harb \& Petre 1999) that would imply the presence of electrons with energies up to $\sim 50 \mathrm{TeV}$ in those regions. The VHE gamma-ray emission from the SS 433/W50 interaction regions was first considered by Aharonian \& Atoyan (1998), who estimated gamma-ray fluxes at a level of $\sim 10^{-12} \mathrm{ph} \mathrm{cm}^{-2} \mathrm{~s}^{-1}$ for the eastern $e 3$ region produced by electrons scattering off CMB photons. Bordas et al. (2009) also considered the non-thermal emission produced in microquasar jets/ISM interaction regions, providing gamma-ray flux estimates as a function of the kinetic power of the jets, age of the system, and particle density of the environment. The application of this model to SS 433/W50 yielded fluxes at the level of $\sim 10^{-13} \mathrm{erg} \mathrm{cm}^{-2} \mathrm{~s}^{-1}$ for $E>$ $250 \mathrm{GeV}$ for an assumed distance to the system of $5.5 \mathrm{kpc}$ (Bordas et al. 2010), which are roughly at the level of the upper limits reported here. However, as noted in Aharonian \& Atoyan (1998; see also discussion in Safi-Harb \& Petre 1999; and Aharonian et al. 2005), electrons accelerated at the interaction region shock interface could lose most of their energy mainly through synchrotron emission for ambient magnetic fields at or above $\sim 10 \mu \mathrm{G}$, preventing an effective channelling through IC scattering that is relevant for the production of gamma rays at high and very high energies. The integral flux upper limits for the interaction regions shown in Table 2, together with the assumption that the same high-energy electron population is responsible for the observed (synchrotron) X-ray emission and the putative gamma-ray fluxes, can be used to constrain the magnetic fields present in the shocked SS 433 jets/ISM interaction regions. Rowell \& HEGRA Collaboration (2001; see also Aharonian et al. 2005) make use of HEGRA upper lim- its obtained for the $e 3$ interaction region $\left(\Phi_{\text {HEGRA }} \leq 2.1 \times\right.$ $10^{-12} \mathrm{ph} \mathrm{cm}^{-2} \mathrm{~s}^{-1}$ ) and the predictions by Aharonian et al. (1997) to derive a lower limit on the post-shock magnetic field in this region of $\sim 13 \mu \mathrm{G}$. Using the upper limits reported here, a more constraining lower limit on the magnetic field of $20-25 \mu \mathrm{G}$ is obtained.

The huge kinetic luminosity and baryonic matter transported by the SS 433 jets and the presence of the surrounding target material provided by the disk wind and/or the W50 nebula render $p p$ interactions at those larger scales a good $\mathrm{TeV}$ emission mechanism as well, as shown for even more modest energy budgets (see e.g. Bosch-Ramon et al. 2005). Bordas et al. (2015) found a gamma-ray signal from the direction of SS 433/W50. The steadiness of the flux and the derived spectral properties, with the gamma-ray emission extending only from $200 \mathrm{MeV}$ to $800 \mathrm{MeV}$, prompted Bordas et al. (2015) to suggest a jet-medium interaction scenario for the observed emission. A cut-off power law was needed to fit the Fermi-LAT spectrum with cut-off energies of a few $\mathrm{GeV}$. The upper limits reported here are therefore fully consistent with the Fermi-LAT extrapolation of the fitted spectra. If the $\mathrm{MeV} / \mathrm{GeV}$ emission is produced by relativistic particles in SS 433 jets, as suggested in Bordas et al. (2015), the acceleration mechanism may be only relatively efficient, thereby preventing a significant detection of the system in the VHE regime.

The upper limits reported here for SS 433 and those obtained on the steady emission from other well-established Galactic microquasars (e.g. Cyg X-1, Cyg X-3, GRS 1915+105, MWC 656, and Cir X-1; see e.g. Nicholas \& Rowell 2008; H.E.S.S. Collaboration 2009; H.E.S.S. Collaboration 2018a; Saito et al. 2009; Aleksić et al. 2010, 2015) imply that if their jets are also baryon loaded as in SS 433 (see also the case of 4U1630-47, Díaz Trigo et al. 2013), their contribution to the Galactic cosmic-ray flux must be limited to relatively low energies in the $\mathrm{GeV}$ domain, as more efficient proton acceleration is constrained by the lack of VHE gamma-ray emission.

Acknowledgements. The authors would like to thank M. M. Reynoso for sharing data and information from his paper Reynoso et al. (2008b). The MAGIC Collaboration would like to thank the Instituto de Astrofísica de Canarias for the excellent working conditions at the Observatorio del Roque de los Muchachos in La Palma. The financial support of the German B.M.B.F. and M.P.G., the Italian INFN and INAF, the Swiss National Fund SNF, the ERDF under the Spanish MINECO (FPA2015-69818-P, FPA2012-36668, FPA201568378-P, FPA2015-69210-C6-2-R, FPA2015-69210-C6-4-R, FPA2015-69210C6-6-R, AYA2015-71042-P, AYA2016-76012-C3-1-P, ESP2015-71662-C2-2-P, CSD2009-00064), and the Japanese JSPS and MEXT is gratefully acknowledged. This work was also supported by the Spanish Centro de Excelencia "Severo Ochoa" SEV-2012-0234 and SEV-2015-0548, and Unidad de Excelencia "María de Maeztu" MDM-2014-0369, by the Croatian Science Foundation (HrZZ) Project 09/176 and the University of Rijeka Project 13.12.1.3.02, by the DFG Collaborative Research Centers SFB823/C4 and SFB876/C3, and by the Polish MNiSzW grant 745/N-HESS-MAGIC/2010/0. The support of the Namibian authorities and the University of Namibia in facilitating the construction and operation of H.E.S.S. is gratefully acknowledged, as is the support by the German Ministry for Education and Research (BMBF), the Max Planck Society, the German Research Foundation (DFG), the French Ministry for Research, the CNRS-IN2P3 and the Astroparticle Interdisciplinary Programme of the CNRS, the UK Science and Technology Facilities Council (STFC), the IPNP of the Charles University, the Czech Science Foundation, the Polish Ministry of Science and Higher Education, the South African Department of Science and Technology and National Research Foundation, and by the University of Namibia. We appreciate the excellent work of the technical support staff in Berlin, Durham, Hamburg, Heidelberg, Palaiseau, Paris, Saclay, and in Namibia in the construction and operation of the equipment.

\section{References}

Aartsen, M. G., Ackermann, M., Adams, J., et al. 2014, ApJ, 796, 109 Abdo, A. A., Allen, B., Berley, D., et al. 2007, ApJ, 664, L91 
Abell, G. O., \& Margon, B. 1979, Nature, 279, 701

Aharonian, F. A., \& Atoyan, A. M. 1998, New Astron. Rev., , 42, 579

Aharonian, F. A., Atoyan, A. M., \& Kifune, T. 1997, MNRAS, 291, 162

Aharonian, F., Akhperjanian, A., Beilicke, M., et al. 2005, A\&A, 439, 635

Aharonian, F., Akhperjanian, A. G., Bazer-Bachi, A. R., et al. 2006a, A\&A, 457, 899

Aharonian, F., Akhperjanian, A. G., Bazer-Bachi, A. R., et al. 2006b, ApJ, 636, 777

Albert, J., Aliu, E., Anderhub, H., \& et al. 2008, Nucl. Instr. Meth. Phys. Res. A, 588,424

Aleksić, J., Antonelli, L. A., Antoranz, P., et al. 2010, ApJ, 721, 843

Aleksić, J., Alvarez, E. A., Antonelli, L. A., et al. 2012a, Astropart. Phys., 35 435

Aleksić, J., Alvarez, E. A., Antonelli, L. A., et al. 2012b, A\&A, 540, A69

Aleksić, J., Ansoldi, S., Antonelli, L. A., et al. 2015, A\&A, 576, A36

Aleksić, J., Ansoldi, S., Antonelli, L. A., et al. 2016, Astropart. Phys., 72, 76

Atoyan, A. M., \& Aharonian, F. A. 1999, MNRAS, 302, 253

Band, D. L., \& Grindlay, J. E. 1986, ApJ, 311, 595

Bernlöhr, K., Carrol, O., Cornils, R., et al. 2003, Astropart. Phys., 20, 111

Blundell, K. M., \& Bowler, M. G. 2004, ApJ, 616, L159

Bordas, P., Bosch-Ramon, V., Paredes, J. M., \& Perucho, M. 2009, A\&A, 497, 325

Bordas, P., Bosch-Ramon, V., \& Paredes, J. M. 2010, Int. J. Mod. Phys. D, 19, 749

Bordas, P., Yang, R., Kafexhiu, E., \& Aharonian, F. 2015, ApJ, 807, L8

Bosch-Ramon, V., \& Khangulyan, D. 2009, Int. J. Mod. Phys. D, 18, 347

Bosch-Ramon, V., Aharonian, F. A., \& Paredes, J. M. 2005, in Astrophysical Sources of High Energy Particles and Radiation, eds. T. Bulik, B. Rudak, \& G. Madejski, AIP Conf. Ser., 801, 196

Bosch-Ramon, V., Romero, G. E., \& Paredes, J. M. 2006, A\&A, 447, 263

Brinkmann, W., Pratt, G. W., Rohr, S., Kawai, N., \& Burwitz, V. 2007, A\&A, 463,611

Cherepashchuk, A. 2002, Sp. Sc. Rev., 102, 23

de Naurois, M., \& Rolland, L. 2009, Astropart. Phys., 32, 231

Díaz Trigo, M., Miller-Jones, J. C. A., Migliari, S., Broderick, J. W., \& Tzioumis, T. 2013, Nature, 504, 260

Dubner, G. M., Holdaway, M., Goss, W. M., \& Mirabel, I. F. 1998, AJ, 116 1842

Eikenberry, S. S., Cameron, P. B., Fierce, B. W., et al. 2001, ApJ, 561, 1027

Fabian, A. C., \& Rees, M. J. 1979, MNRAS, 187, 13P

Fabrika, S. 2004, Astrophys. Space Phys. Rev., 12, 1

Fabrika, S. N. 1993, MNRAS, 261, 241

Fabrika, S. N., \& Sholukhova, O. 2008, in Microquasars and Beyond, 52

Farnes, J. S., Gaensler, B. M., Purcell, C., et al. 2017, MNRAS, 467, 4777

Fomin, V. P., Stepanian, A. A., Lamb, R. C., et al. 1994, Astropart. Phys., 2, 137

Fuchs, Y. 2002, ArXiv e-prints [arXiv: astro-ph/0207429]

Fuchs, Y., Koch Miramond, L., \& Ábrahám, P. 2006, A\&A, 445, 1041

Gies, D. R., McSwain, M. V., Riddle, R. L., et al. 2002, ApJ, 566, 1069

Goodall, P. T., Alouani-Bibi, F., \& Blundell, K. M. 2011, MNRAS, 414, 2838

Goranskij, V. 2011, Peremennye Zvezdy, 31, 5

Green, D. W. E. 2006, Cavendish Laboratory (Cambridge, UK), 8661, 2

Gregory, P. C., Scott, W. K., Douglas, K., \& Condon, J. J. 1996, ApJS, 103, 427

Guenette, R., \& for the VERITAS Collaboration 2009, ArXiv e-prints [arXiv: 0908.0714]

Hayashi, S., Kajino, F., Naito, T., et al. 2009, Astropart. Phys., 32, 112

Heinz, S., \& Sunyaev, R. 2002, A\&A, 390, 751

H.E.S.S. Collaboration, Acero, F., Aharonian, F., et al. 2009, A\&A, 508, 1135

H.E.S.S. Collaboration (Abdalla, H., Abramowski, A., et al.) 2018a, A\&A, 612, A10 (H.E.S.S. SI)

H.E.S.S. Collaboration (Abdalla, H., Abramowski, A., et al.) 2018b, A\&A, 612, A2 (H.E.S.S. SI)

Hillas, A. M. 1985, International Cosmic Ray Conference, 3, 445

Kaufman Bernadó, M. M., Romero, G. E., \& Mirabel, I. F. 2002, A\&A, 385, L10

Kotani, T., Kawai, N., Aoki, T., et al. 1994, PASJ, 46, L147

Levinson, A., \& Blandford, R. 1996, ApJ, 456, L29

Lockman, F. J., Blundell, K. M., \& Goss, W. M. 2007, MNRAS, 381, 881

Margon, B. 1984, ARA\&A, 22, 507

Margon, B., \& Anderson, S. F. 1989, ApJ, 347, 448

Marshall, H. L., Canizares, C. R., \& Schulz, N. S. 2002, ApJ, 564, 941

Migliari, S., Fender, R., \& Méndez, M. 2002, Science, 297, 1673

Moldowan, A., \& Safi-Harb, S. 2005, JRASC, 99, 141

Nicholas, B., \& Rowell, G. 2008, in eds. F. A. Aharonian, W. Hofmann, \& F. Rieger, AIP Conf. Ser., 1085, 245

Orellana, M., Bordas, P., Bosch-Ramon, V., Romero, G. E., \& Paredes, J. M. 2007, A\&A, 476, 9
Reynoso, M. M., Christiansen, H. R., \& Romero, G. E. 2008a, Astropart. Phys., 28, 565

Reynoso, M. M., Romero, G. E., \& Christiansen, H. R. 2008b, MNRAS, 387, 1745

Rolke, W. A., López, A. M., \& Conrad, J. 2005, Nucl. Instr. Meth. Phys. Res. A, 551,493

Rowell, G. P., \& HEGRA Collaboration. 2001, ArXiv eprints [arXiv: astro-ph/0104288]

Safi-Harb, S., \& Ögelman, H. 1997, ApJ, 483, 868

Safi-Harb, S., \& Petre, R. 1999, ApJ, 512, 784

Saito, T. Y., Zanin, R., Bordas, P., et al. 2009, ArXiv eprints [arXiv:0907 . 1017]

Torres, D. F. \& Reimer, A. 2011, A\&A, 528, L2

Yamamoto, H., Ito, S., Ishigami, S., et al. 2008, PASJ, 60, 715

Zealey, W. J., Dopita, M. A., \& Malin, D. F. 1980, MNRAS, 192, 731

Zwitter, T., Calvani, M., \& D'Odorico, S. 1991, A\&A, 251, 92

ETH Zurich, 8093 Zurich, Switzerland

Università di Udine, and INFN Trieste, 33100 Udine, Italy

3 INAF National Institute for Astrophysics, 00136 Rome, Italy

Università di Padova and INFN, 35131 Padova, Italy

5 Croatian MAGIC Consortium, Rudjer Boskovic Institute, University of Rijeka, University of Split - FESB, University of Zagreb - FER, University of Osijek, 10000 Zagreb, Croatia

6 Saha Institute of Nuclear Physics, 1/AF Bidhannagar, Salt Lake, Sector-1, 700064 Kolkata, India

7 Max-Planck-Institut für Physik, 80805 München, Germany

8 Universidad Complutense, 28040 Madrid, Spain

9 Inst. de Astrofísica de Canarias, 38200 La Laguna, Tenerife, Spain

10 Universidad de La Laguna, Dpto. Astrofísica, 38206 La Laguna, Tenerife, Spain

11 University of Łódź, 90236 Lodz, Poland

12 Deutsches Elektronen-Synchrotron (DESY), 15738 Zeuthen, Germany

13 Institut de Fisica d'Altes Energies (IFAE), The Barcelona Institute of Science and Technology, Campus UAB, 08193 Bellaterra (Barcelona), Spain

14 Università di Siena, and INFN Pisa, 53100 Siena, Italy

15 Institute for Space Sciences (CSIC/IEEC), 08193 Barcelona, Spain

16 Technische Universität Dortmund, 44221 Dortmund, Germany

17 Universität Würzburg, 97074 Würzburg, Germany

18 Finnish MAGIC Consortium, Tuorla Observatory, University of Turku and Astronomy Division, University of Oulu, 90014 Oulu, Finland

19 Unitat de Física de les Radiacions, Departament de Física, and CERES-IEEC, Universitat Autònoma de Barcelona, 08193 Bellaterra, Spain

20 Universitat de Barcelona, ICC, IEEC-UB, 08028 Barcelona, Spain

21 Japanese MAGIC Consortium, ICRR, The University of Tokyo, Department of Physics and Hakubi Center, Kyoto University, Tokai University, The University of Tokushima, 770-8501 Tokushima, Japan

22 Inst. for Nucl. Research and Nucl. Energy, 1784 Sofia, Bulgaria

23 Università di Pisa, and INFN Pisa, 56126 Pisa, Italy

24 ICREA and Institute for Space Sciences (CSIC/IEEC), 08193 Barcelona, Spain

25 Also at the Department of Physics of Kyoto University, 606-8501 Kyoto, Japan

26 Now at Centro Brasileiro de Pesquisas Físicas (CBPF/MCTI), R. Dr. Xavier Sigaud, 150 - Urca, Rio de Janeiro - RJ, 22290-180, Brazil

27 Now at NASA Goddard Space Flight Center, Greenbelt, MD 20771, USA

28 Department of Physics and Department of Astronomy, University of Maryland, College Park, MD 20742, USA

29 Institut für Physik, Humboldt-Universität zu Berlin, Newtonstr. 15, 12489 Berlin, Germany

30 Also at University of Trieste

31 Also at Japanese MAGIC Consortium 
32 Now at Finnish Centre for Astronomy with ESO (FINCA), Turku, Finland

33 Also at INAF-Trieste and Dept. of Physics \& Astronomy, University of Bologna, 40126 Bologna, Italy

34 Now at Laboratoire AIM (UMR 7158 CEA/DSM, CNRS, Université Paris Diderot), Irfu/Service d'Astrophysique, CEA-Saclay, 91191 Gif-sur-Yvette Cedex, France

35 Now at INAF/IAPS-Roma, 00133 Roma, Italy; Centre for Space Research, North-West University, 2520 Potchefstroom, South Africa

${ }^{36}$ Universität Hamburg, Institut für Experimentalphysik, Luruper Chaussee 149, 22761 Hamburg, Germany

37 Max-Planck-Institut für Kernphysik, PO Box 103980, 69029 Heidelberg, Germany

38 Dublin Institute for Advanced Studies, 31 Fitzwilliam Place, Dublin 2, Ireland

39 National Academy of Sciences of the Republic of Armenia, Marshall Baghramian Avenue, 24, 0019 Yerevan, Republic of Armenia

40 Yerevan Physics Institute, 2 Alikhanian Brothers St., 375036 Yerevan, Armenia

41 Institut für Physik, Humboldt-Universität zu Berlin, Newtonstr. 15, 12489 Berlin, Germany

42 University of Namibia, Department of Physics, 13301 Private Bag, Windhoek, Namibia

43 GRAPPA, Anton Pannekoek Institute for Astronomy, University of Amsterdam, Science Park 904, 1098 XH Amsterdam, The Netherlands

44 Department of Physics and Electrical Engineering, Linnaeus University, 35195 Växjö, Sweden

45 Institut für Theoretische Physik, Lehrstuhl IV: Weltraum und Astrophysik, Ruhr-Universität Bochum, 44780 Bochum, Germany

46 GRAPPA, Anton Pannekoek Institute for Astronomy and Institute of High-Energy Physics, University of Amsterdam, Science Park 904, 1098 XH Amsterdam, The Netherlands

${ }^{47}$ Institut für Astro- und Teilchenphysik, Leopold-Franzens-Universität Innsbruck, 6020 Innsbruck, Austria

48 School of Physical Sciences, University of Adelaide, 5005 Adelaide, Australia

49 LUTH, Observatoire de Paris, PSL Research University, CNRS, Université Paris Diderot, 5 place Jules Janssen, 92190 Meudon, France

50 Sorbonne Universités, UPMC Université Paris 06, Université Paris Diderot, Sorbonne Paris Cité, CNRS, Laboratoire de Physique

Nucléaire et de Hautes Energies (LPNHE), 4 place Jussieu, 75252 Paris Cedex 5, France

51 Laboratoire Univers et Particules de Montpellier, Université Montpellier, CNRS/IN2P3, CC 72, Place Eugène Bataillon, 34095 Montpellier Cedex 5, France

52 DSM/Irfu, CEA Saclay, 91191 Gif-Sur-Yvette Cedex, France

53 Astronomical Observatory, The University of Warsaw, Al. Ujazdowskie 4, 00-478 Warsaw, Poland

54 Aix-Marseille Université, CNRS/IN2P3, CPPM UMR 7346, 13288 Marseille, France

55 Instytut Fizyki Jạdrowej PAN, ul. Radzikowskiego 152, 31-342 Kraków, Poland
${ }^{56}$ Funded by EU FP7 Marie Curie, grant agreement No. PIEF-GA-2012-332350

57 School of Physics, University of the Witwatersrand, 1 Jan Smuts Avenue, Braamfontein, 2050 Johannesburg, South Africa

58 Laboratoire d'Annecy-le-Vieux de Physique des Particules, Université Savoie Mont-Blanc, CNRS/IN2P3, 74941 Annecy-le-Vieux, France

59 Landessternwarte, Universität Heidelberg, Königstuhl, 69117 Heidelberg, Germany

${ }^{60}$ Université Bordeaux, CNRS/IN2P3, Centre d'Études Nucléaires de Bordeaux Gradignan, 33175 Gradignan, France

61 Oskar Klein Centre, Department of Physics, Stockholm University, Albanova University Center, 10691 Stockholm, Sweden

62 Wallenberg Academy Fellow

63 Institut für Astronomie und Astrophysik, Universität Tübingen, Sand 1, 72076 Tübingen, Germany

${ }^{64}$ Laboratoire Leprince-Ringuet, École Polytechnique, CNRS/IN2P3, 91128 Palaiseau, France

65 APC, AstroParticule et Cosmologie, Université Paris Diderot, CNRS/IN2P3, CEA/Irfu, Observatoire de Paris, Sorbonne Paris Cité, 10 rue Alice Domon et Léonie Duquet, 75205 Paris Cedex 13, France

${ }^{66}$ Univ. Grenoble Alpes, IPAG, 38000 Grenoble, France; CNRS, IPAG, 38000 Grenoble, France

67 Department of Physics and Astronomy, The University of Leicester, University Road, Leicester, LE1 7RH, UK

68 Nicolaus Copernicus Astronomical Center, Polish Academy of Sciences, ul. Bartycka 18, 00-716 Warsaw, Poland

69 Institut für Physik und Astronomie, Universität Potsdam, Karl-Liebknecht-Strasse 24/25, 14476 Potsdam, Germany

${ }^{70}$ Friedrich-Alexander-Universität Erlangen-Nürnberg, Erlangen Centre for Astroparticle Physics, Erwin-Rommel-Str. 1, 91058 Erlangen, Germany

71 DESY, 15738 Zeuthen, Germany

72 Obserwatorium Astronomiczne, Uniwersytet Jagielloński, ul. Orla 171, 30-244 Kraków, Poland

73 Centre for Astronomy, Faculty of Physics, Astronomy and Informatics, Nicolaus Copernicus University, Grudziadzka 5, 87-100 Torun, Poland

74 Department of Physics, University of the Free State, PO Box 339, 9300 Bloemfontein, South Africa

75 Heisenberg Fellow (DFG), ITA Universität Heidelberg, Germany

76 GRAPPA, Institute of High-Energy Physics, University of Amsterdam, Science Park 904, 1098 XH Amsterdam, The Netherlands

77 Department of Physics, Rikkyo University, 3-34-1 Nishi-Ikebukuro, Toshima-ku, 171-8501 Tokyo, Japan

78 Japan Aerpspace Exploration Agency (JAXA), Institute of Space and Astronautical Science (ISAS), 3-1-1 Yoshinodai, Chuo-ku, Sagamihara, 229-8510 Kanagawa, Japan

79 Now at Santa Cruz Institute for Particle Physics and Department of Physics, University of California at Santa Cruz, Santa Cruz, CA 95064, USA

80 Department of Physics and Astronomy, University of Manitoba, Winnipeg, MB R3T 2N2, Canada 